\title{
Brief Announcement: Semantics of Eventually Consistent Replicated Sets *
}

\author{
Annette Bieniusa $^{1}$, Marek Zawirski ${ }^{1,2}$, Nuno Preguiça ${ }^{3,1}$, Marc Shapiro ${ }^{1}$, \\ Carlos Baquero ${ }^{4}$, Valter Balegas ${ }^{3}$, and Sérgio Duarte ${ }^{3}$ \\ 1 INRIA/LIP6, Paris, France \\ 2 UPMC, Paris, France \\ 3 CITI, Universidade Nova de Lisboa, Portugal \\ 4 HASLab, INESC Tec and Universidade do Minho, Portugal
}

This paper studies the semantics of sets under eventual consistency. The set is a pervasive data type, used either directly or as a component of more complex data types, such as maps or graphs. Eventual consistency of replicated data supports concurrent updates, reduces latency and improves fault tolerance, but forgoes strong consistency (e.g., linearisability). Accordingly, several cloud computing platforms implement eventually-consistent replicated sets [24].

The sequential semantics of a set are well known, and are defined by individual updates, e.g., $\{$ true $\} a d d(e)\{e \in S\}$ (in " $\{$ pre-condition $\}$ computation \{post-condition\}" notation), where $S$ denotes its abstract state. However, the semantics of concurrent modifications is left underspecified or implementationdriven.

We propose the following Principle of Permutation Equivalence to express that concurrent behaviour conforms to the sequential specification: "If all sequential permutations of updates lead to equivalent states, then it should also hold that concurrent executions of the updates lead to equivalent states." It implies the following behavior, for some updates $u$ and $u^{\prime}$ :

$$
\{P\} u ; u^{\prime}\{Q\} \wedge\{P\} u^{\prime} ; u\left\{Q^{\prime}\right\} \wedge Q \Leftrightarrow Q^{\prime} \Rightarrow\{P\} u \| u^{\prime}\{Q\}
$$

Specifically for replicated sets, the Principle of Permutation Equivalence requires that $\{e \neq f\}$ add $(e) \|$ remove $(f)\{e \in S \wedge f \notin S\}$, and similarly for operations on different elements or idempotent operations. Only the pair add(e)\| remove $(e)$ is unspecified by the principle, since $\operatorname{add}(e) ;$ remove $(e)$ differs from remove $(e) ; \operatorname{add}(e)$. Any of the following post-conditions ensures a deterministic result:

$$
\begin{array}{cc}
\left\{\perp_{e} \in S\right\} & - \text { Error mark } \\
\{e \in S\} & - \text { add wins } \\
\{e \notin S\} & - \text { remove wins } \\
\left\{\operatorname{add}(e)>_{\text {CLK }} \text { remove }(e) \Leftrightarrow e \in S\right\} & \text { - Last Writer Wins }(\text { LWW })
\end{array}
$$

where $<$ CLK compares unique clocks associated with the operations. Note that

\footnotetext{
* This research is supported in part by ANR project ConcoRDanT (ANR-10-BLAN 0208), by ERDF, COMPETE Programme, by Google European Doctoral Fellowship in Distributed Computing received by Marek Zawirski, and FCT projects \#PTDC/EIA-EIA/104022/2008 and \#PTDC/EIA-EIA/108963/2008.
} 


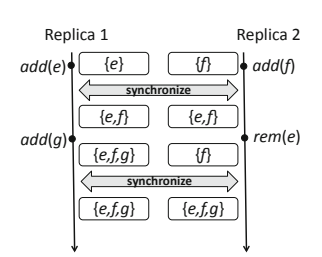

(a) Dynamo shopping

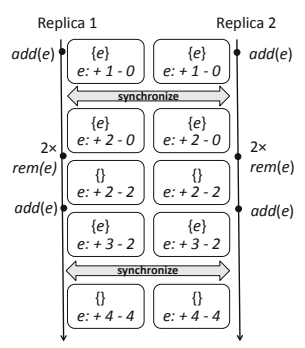

(b) C-Set

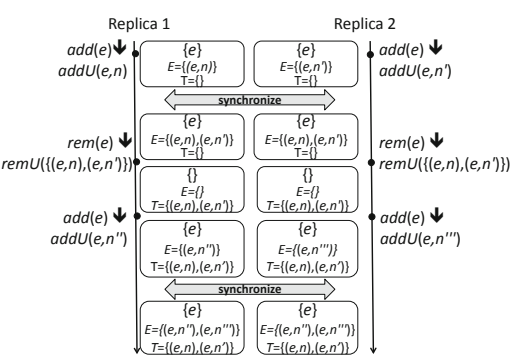

(c) OR-Set cart

Fig. 1. Examples of anomalies and a correct design

not all concurrency semantics can be explained as a sequential permutation; for instance no sequential execution ever results in an error mark.

A Study of Existing Replicated Set Designs. In the past, several designs have been proposed for maintaining a replicated set. Most of them violate the Principle of Permutation Equivalence (Fig. 1). For instance, the Amazon Dynamo shopping cart [2] is implemented using a register supporting read and write (assignment) operations, offering the standard sequential semantics. When two writes occur concurrently, the next read returns their union. As noted by the authors themselves, in case of concurrent updates even on unrelated elements, a remove may be undone (Fig. 1(a)].

Sovran et al. and Asian et al. 411] propose a set variant, C-Set, where for each element the associated add and remove updates are counted. The element is in the abstraction if their difference is positive. C-Set violates the Principle of Permutation Equivalence (Fig. 1(b)]. When delivering the updates to both replicas as sketched, the add and remove counts are equal, i.e., $e$ is not in the abstraction, even though the last update at each replica is add(e).

Shapiro et al. propose a replicated set design, called OR-Set, [3] that ensures that concurrent add/remove operations commute. Unlike the others, it satisfies the Principle of Permutation Equivalence, as illustrated in Figure 1(c). Hidden unique tokens distinguish between different invocations of add, making it possible to to precisely track which add operations are affected by a remove.

\section{References}

1. Aslan, K., Molli, P., Skaf-Molli, H., Weiss, S.: C-Set: a commutative replicated data type for semantic stores. In: Int. W. on REsource Discovery, RED (2011)

2. DeCandia, G., Hastorun, D., et al.: Dynamo: Amazon's highly available key-value store. In: Symp. on Op. Sys. Principles, SOSP (2007)

3. Shapiro, M., Preguiça, N., Baquero, C., Zawirski, M.: Conflict-Free Replicated Data Types. In: Défago, X., Petit, F., Villain, V. (eds.) SSS 2011. LNCS, vol. 6976, pp. 386-400. Springer, Heidelberg (2011)

4. Sovran, Y., Power, R., Aguilera, M.K., Li, J.: Transactional storage for georeplicated systems. In: Symp. on Op. Sys. Principles, SOSP (2011) 